\title{
KAMRA corneal inlay for the correction of preshyopia
}

\author{
Ioannis A. Mallias, Panagiota Mylova, Anastasia Tassiopoulou, Ronis Christidis, Aikaterini Mouzaka \\ Laser Plus Eye, Nea Smyrni, Athens, Greece
}

\begin{abstract}
This is a case report of two patients who underwent presbyopia correction with KAMRA corneal inlay (KAMRA; AcuFocus, Irvine, CA) combined with LASIK surgery. The surgical technique is thoroughly explained, as well as the advantages and disadvantages of the procedure. In both patients, near uncorrected visual acuity significantly improved and distance uncorrected visual acuity remained unchanged. There were no changes in the corneal topography after the corneal inlay implantation.
\end{abstract}

KEY WORDS: presbyopia, surgery, corneal inlay implantation

Ophthalmol J 2017; Vol. 2, No. 1, 28-32

\section{INTRODUCTION}

Through the years, many methods have been tried in order to treat presbyopia. Some of them are: multifocal intraocular lenses [1], monovision with intraocular lenses or LASIK [2], and implantation of presbyopia-correcting intraocular lenses [3], but none of them is without disadvantages, which usually lead to patient dissatisfaction.

Corneal inlays have been studied for over 40 years. The first inlays were made from donor corneal tissue shaped by cryolathe [4]. Later, various materials were employed to develop synthetic corneal inlays such as Plexiglas, PMMA, etc. However, these ingredients were associated with stromal necrosis and extrusion of the implant [5].

Hydrogel implants were found to be permeable to water and solutes, which helped the cornea take its necessary nutrients, but they opacified due to impregnation with proteins. Some corneas also underwent necrosis due to the thickness of these inlays [6]. Over the years, hydrogels underwent more development [7]. Further modification and improvement in the design of the inlays is being investigated in order to achieve better tolerance in the human cornea. These modifications mostly include decrease in inlay thickness, use of biocompatible materials, incorporation of holes on the inlay in order to facilitate nutrients reach the cornea, and optimisation of the depth of placement in the cornea. Currently there are three types of corneal inlays:

- refractive corneal inlays;

- corneal reshaping inlays;

- small aperture inlays.

Corneal inlays are also an upcoming solution for presbyopia correction, especially the KAMRA inlay, which is a small aperture corneal inlay that is placed into the corneal stroma and blocks the non-focused rays of light, allowing only the focused rays to reach the retina, resulting in an increase of the depth of field [8].

The thickness [9] of KAMRA inlay is $5 \mu \mathrm{m}$ and its aperture [9] is $1.6 \mathrm{~mm}$, which is the optimal size for improving near vision and maintaining distance vision. It is inserted into the cornea through a "pocket" that is made by the surgeon with a femtosecond laser. The inlay must be implanted into the non-dominant eye of the patient, and it has many tiny holes in order to allow corneal nutrition. The 
inlay can be implanted between 200 and $250 \mu \mathrm{m}$ corneal depth.

The ideal depth, however, is $240 \mu \mathrm{m}$ in order not to induce changes in corneal topography. The advantages of the KAMRA inlay are that near and intermediate vision are significantly improved, with only mild deterioration of distance vision. The procedure is not as invasive as other techniques, and it is reversible [10]. This presbyopia correction technique also has the advantage of leaving the crystalline lens intact, and if it is needed it can be combined with refractive surgery [9]. The procedure can also be done in patients who have already undergone refractive surgery. If the patient has already undergone a LASIK surgery, then the inlay should be implanted at least $100 \mu \mathrm{m}$ below the prior LASIK flap. There are studies [11] showing that the KAMRA inlay is well tolerated in the long term. Also, the postoperative distance visual acuity is not influenced by the pupil size [12] of the patient after KAMRA inlay implantation. There are also long-term studies [13-15] that show a significant increase of visual acuity in intermediate and near vision [16], but with a slight decrease in distance vision, in the eye that the inlay is implanted. However, binocular distance vision and stereopsis do not seem to be affected by the procedure [16].

The inclusion criteria [9] for such a procedure are: 1 . patient's age between 45-65 years; and 2. patient's refractive error should be between $-5.00 \mathrm{D}$ and +3.00 D with cylinder, no more than $3.00 \mathrm{D}$. If the patient develops cataracts, the cataract procedure can be done without the need of removing the inlay. Optimal results in vision are reached if the refraction in the recessive eye is $-0.75 \mathrm{D}$ and in the dominant eye is plano.

\section{CASE REPORT PATIENT 1}

A female, 48 years old, with best corrected distance visual acuity (BCDVA) 20/20 on both eyes (refraction OD: +1.00-0.50 × 125 OS: +0.50), underwent presbyopia correction with KAMRA inlay by Dr. Mallias. The patient was thoroughly screened by Dr. Mallias. The KAMRA inlay was implanted in the right eye, which was the non-dominant eye.

Excimer Laser Amaris 750s (Schwind, Germany) and FEMTO LDV Z4 (Ziemer Ophthalmic Systems AG, Switzerland) were employed in order to make the right eye slightly myopic (target: $-0.75 \mathrm{D}$ ) and create a pocket for the inlay, on the same day.
The pocket was created with a $4 \mu \mathrm{m} \times 4 \mu \mathrm{m}$ spot line setting at a depth of $250 \mu \mathrm{m}$.

The first step of the procedure was the creation of the corneal stromal "pocket" for the inlay. The second step of the procedure was the creation of a LASIK flap with the femtosecond laser. The thickness of the flap was $110 \mu \mathrm{m}$. The flap was lifted and hyperopic excimer laser ablation was applied on the corneal stroma. After flap repositioning, the surgeon waited two minutes for the flap to adhere to the corneal stroma. The next step of the procedure was the insertion of the KAMRA inlay in the corneal pocket $(250 \mu \mathrm{m})$ and centration of the inlay based on the first Purkinje image.

Postoperatively the patient was on ofloxacin and dexamethasone four times a day, and preservative-free artificial tears every two hours for one week. After the end of the first postoperative week, the patient was on Loteprednol drops four times a day and preservative-free artificial tears every two hours. Loteprednol was slowly tapered during the first three postoperative months.

At the end of the first postoperative month the uncorrected distance visual acuity (UDVA) of the right eye was 20/25. The uncorrected near visual acuity (UNVA) was J2. At the end of the follow-up period (24 months) distance and near visual acuity remained unchanged and the cornea was clear, with no signs of inflammation.

In Figure 1 the treatment plan of the LASIK surgery is depicted. The ablation zone was $7.79 \mathrm{~mm}$, and the maximum ablation depth was $34.75 \mu \mathrm{m}$.

\section{PATIENT 2}

A male, 49 years old, also underwent presbyopia correction with KAMRA inlay. UDVA was 20/20 on both eyes. The patient was thoroughly screened by Dr. Mallias, and the right eye was found to be the non-dominant eye. The surgeon then proceeded with Femto LASIK surgery on the right eye in order to induce -0.75 dioptres of myopia. Excimer Laser Amaris 750s and FEMTO LDV Z4 were employed in this case as well.

In Figure 2 the LASIK treatment plan of Patient 2 is depicted. The ablation zone was $7.66 \mathrm{~mm}$, and the maximum ablation depth was $19.46 \mu \mathrm{m}$.

The pocket for the KAMRA inlay was created four weeks after LASIK surgery. The settings and the depth of the pocket creation were the same as for Patient 1.

Postoperatively the patient was on ofloxacin and dexamethasone four times a day, and preservative-free artificial tears every two hours for one week. After the 


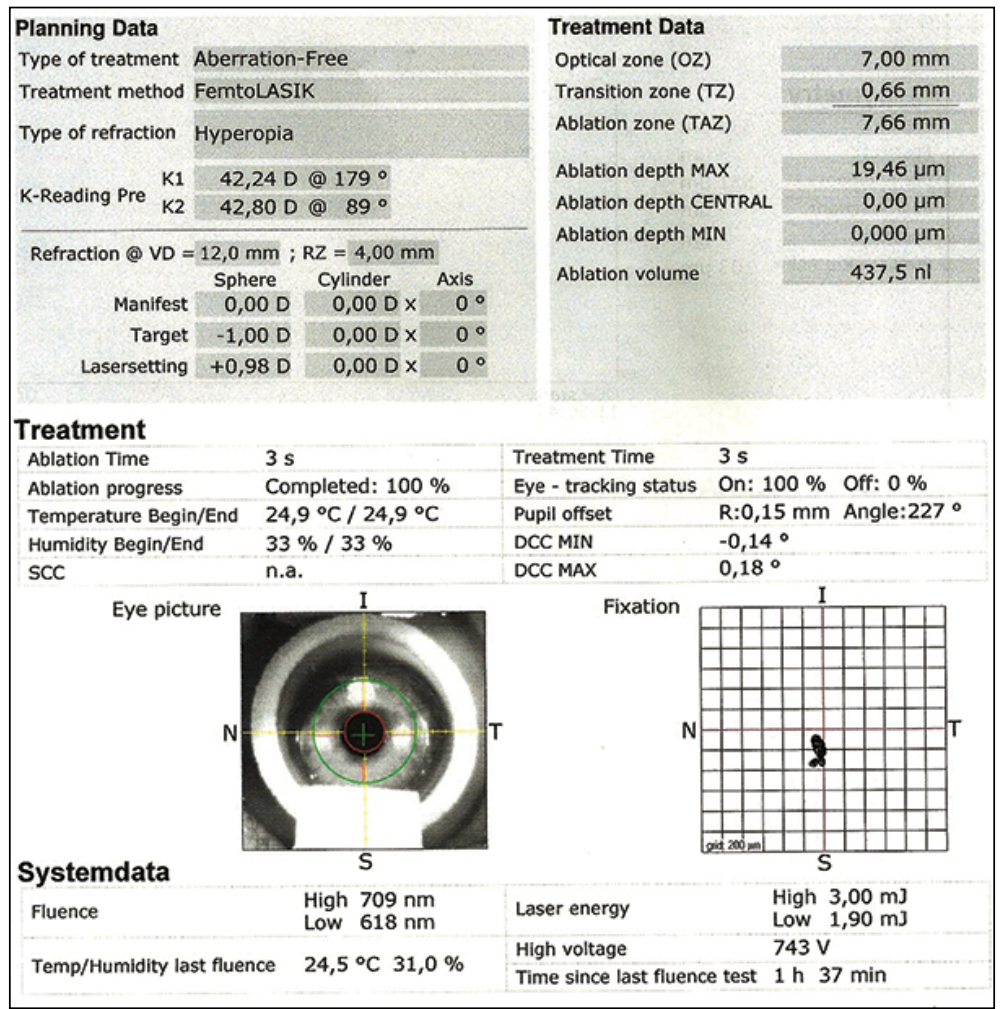

FIGURE 1. Treatment plan of the first patient (female 48 years old)

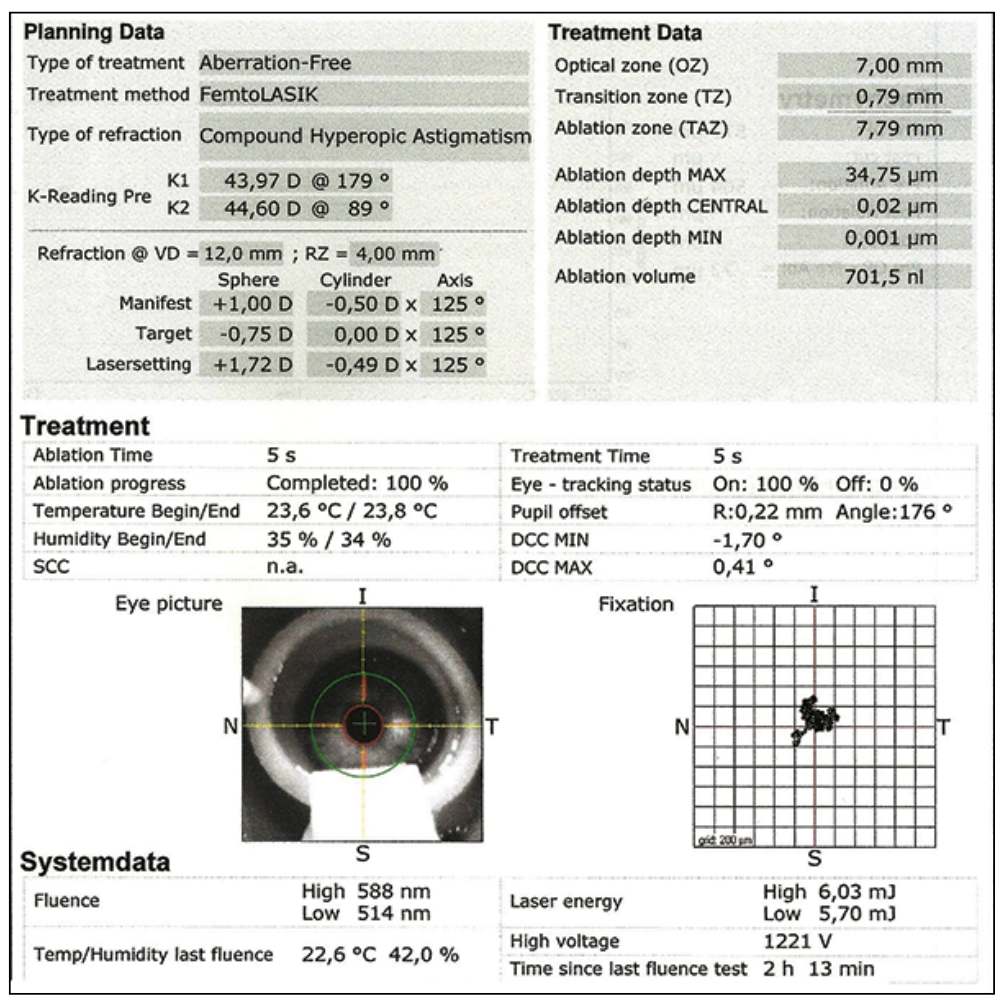

FIGURE 2. Treatment plan of the second patient (male 49 years old) 


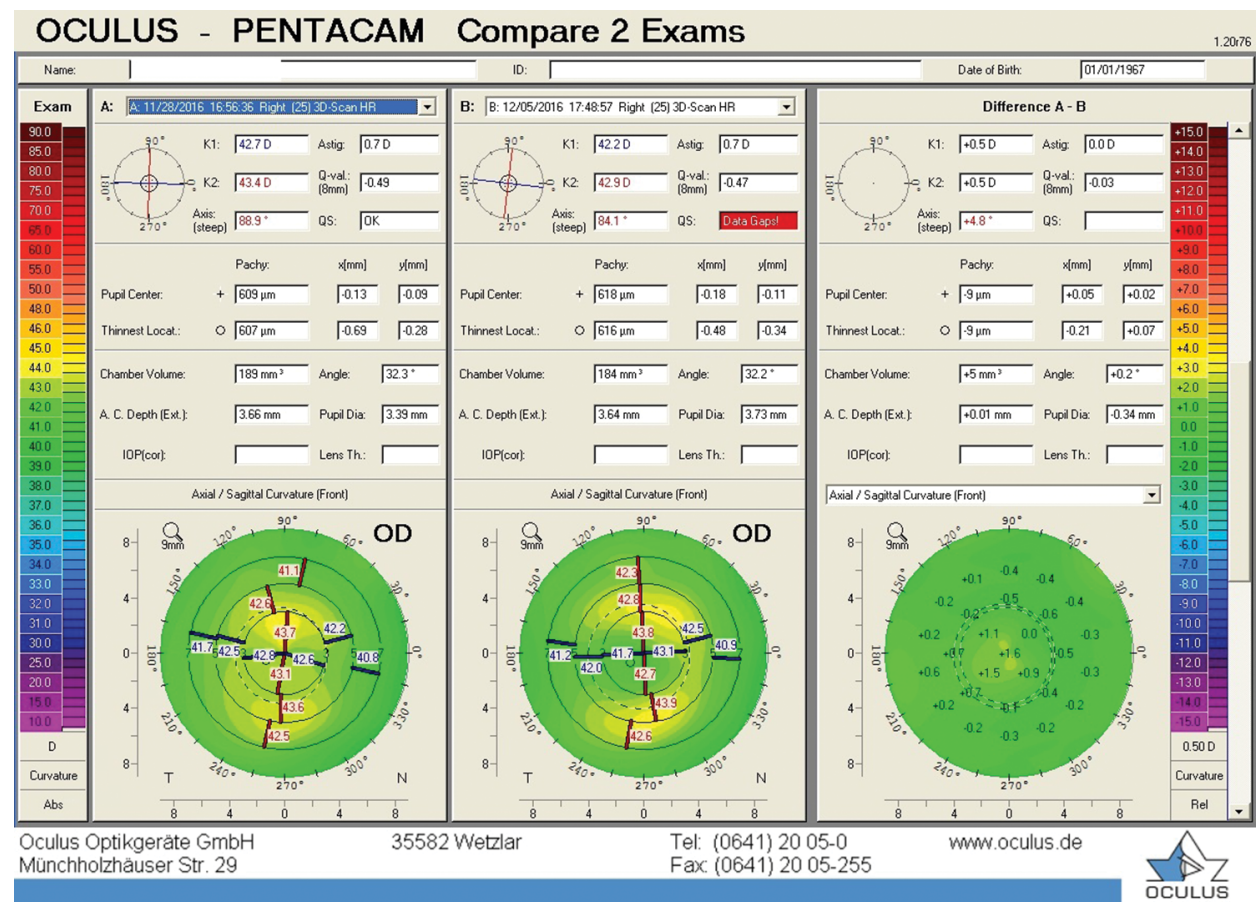

FIGURE 3. Comparison of post-Lasik topography with the post-KAMRA topography of the same patient

end of the first postoperative week, the patient was on Loteprednol drops four times a day and preservative-free artificial tears every two hours. Loteprednol was slowly tapered during the first three postoperative months. His UDVA was 20/25. His UNVA was $\mathrm{J} 2$ one month postoperatively.

\section{DISCUSSION}

Patient 1, developed corneal oedema postoperatively, which lasted for the first three postoperative days. This was attributed to the great stress of the cornea due to the combined surgery. Distance visual acuity (DVA) returned to $20 / 25$ in the right eye one week postoperatively. Patient 2 underwent the two procedures four weeks apart, in order to avoid the corneal stress. This decision resulted in less corneal oedema postoperatively and the patient's DVA was 20/25 from the third postoperative day.

The age group that is interested in the KAMRA inlay (45-65 years old) tends to show hyperopic swift as years go by, and that is why we prefer them to be slightly myopic by the time the inlay is implanted.

Both patients were examined with the Acutarget HD [17] (Visiometrics), which is an instrument evaluating the quality of vision, including: objective scatter index (OSI), assessment of the tear film quality, and measurement of pseudo accommodation. Acutarget also measures the distance between the first Purkinje reflex and the pupil centre: if this distance is less than $300 \mu \mathrm{m}$ then the inlay should be centred to the first Purkinje reflex. If the distance is $300-600 \mu \mathrm{m}$, then the inlay should be centred in the middle of the distance between the pupil centre and the first Purkinje reflex. If the distance is above $600 \mu \mathrm{m}$, then the patient is not a good candidate for the KAMRA procedure. If the measurements with the Acutarget are not optimal, then the inlay implantation should be avoided.

Preoperatively, on the first postoperative day and at the one-month check-up, a Pentacam HR (Oculus, Germany) topography was performed in both patients. It was noticed that the topography has not changed despite the implantation of the corneal inlay. This is attributed to the fact that the inlay is very thin $(5 \mu \mathrm{m})$ and the implantation was deep enough in the corneal layers. In Figure 3 a comparative map is depicted, comparing the post-LASIK topography of the second patient referred to in this report with the post-KAMRA topography of the same patient. The changes are trivial. What should also be noted is that the inlay does not affect the patient's visual fields.

A surgeon who attempts to implant a KAMRA inlay for the first time should be very meticulous with the inlay centration. This is the most demanding part of the procedure.

None of the patients mentioned in this case report had developed epithelial ingrowth. This is 


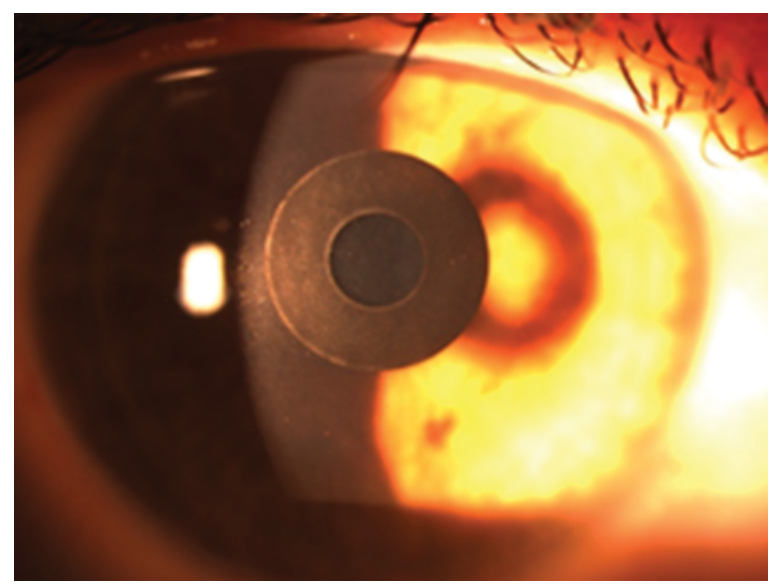

FIGURE 4. KAMRA inlay implanted inside the patient's corneal stroma

probably due to the "lift" of the pocket's verge that the surgeon does in order to insert the inlay inside the pocket. Also, none of the patients mentioned in this report presented any late inlay dislocation. Figure 4 shows the KAMRA inlay implanted inside a patient's cornea. This is the eye of Patient 2.

What should really be taken into consideration is the treatment of pre-existing blepharitis and dry eye. The treatment plays a crucial role in order to achieve good visual outcome and patient satisfaction.

This procedure is appropriate for active people who deal with a lot of intermediate and close distance tasks, who struggle with presbyopia. Some of the patients report that they can read really well without glasses. Others need accessory reading glasses for studying for many hours with a small size font. It should be noted that before proceeding in any surgery, the patient should have been thoroughly evaluated and he/she should have realistic expectations about the procedure's results in order to be satisfied with the outcome.

\section{REFERENCES}

1. Charman WN. Developments in the correction of presbyopia II: surgical approaches. Ophthalmic Physiol Opt. 2014; 34(4): 397-426, doi: 10.1111/opo.12129, indexed in Pubmed: 24716827.
2. Zhang T, Sun Y, Weng S, et al. Aspheric Micro-monovision LASIK in Correction of Presbyopia and Myopic Astigmatism: Early Clinical Outcomes in a Chinese Population. J Refract Surg. 2016; 32(10): 680-685, doi: 10.3928/1081597X-20160628-01, indexed in Pubmed: 27722755.

3. Gibbons A, Ali TK, Waren DP, et al. Causes and correction of dissatisfaction after implantation of presbyopia-correcting intraocular lenses. Clin Ophthalmol. 2016; 10: 1965-1970, doi: 10.2147/0PTH. S114890, indexed in Pubmed: 27784985.

4. Barraquer JI. Modification of refraction by means of intracorneal inclusions. Int Ophthalmol Clin. 1966; 6(1): 53-78, indexed in Pubmed: 5334910.

5. Lane $S L$, Lindstrom RL, Cameron JD, et al. Polysulfone corneal lenses. J Cataract Refract Surg. 1986; 12(1): 50-60, indexed in Pubmed: 3083093

6. Dohlman CH, Refojo MF, Rose J. Synthetic polymers in corneal surgery. I. Glyceryl methacrylate. Arch Ophthalmol. 1967; 77(2): 252-257, indexed in Pubmed: 6019022.

7. Werblin TP, Peiffer RL, Binder PS, et al. Eight years experience with Permalens intracorneal lenses in nonhuman primates. Refract Corneal Surg. 1992; 8(1): 12-22, indexed in Pubmed: 1554634.

8. Dexl AK, Seyeddain 0, Riha W, et al. Reading performance after implantation of a small-aperture corneal inlay for the surgical correction of presbyopia: Two-year follow-up. J Cataract Refract Surg. 2011; 37(3): 525-531, doi: 10.1016/j.jcrs.2010.10.044, indexed in Pubmed: 21262559.

9. https://kamra.com/kamra-inlay/.

10. Jalali S, Aus der Au W, Shaarawy T. AcuFocus Corneal Inlay to Correct Presbyopia Using Femto-LASIK. One Year Results of a Prospective Cohort Study. Klin Monbl Augenheilkd. 2016; 233(4): 360-364, doi: 10.1055/s-0041-111804, indexed in Pubmed: 27116483.

11. Abbouda A, Javaloy J, Alió JL. Confocal microscopy evaluation of the corneal response following AcuFocus KAMRA inlay implantation. J Refract Surg. 2014; 30(3): 172-178, doi: 10.3928/1081597X20140217-04, indexed in Pubmed: 24763721.

12. Tomita M, Kanamori T, Waring GO, et al. Retrospective evaluation of the influence of pupil size on visual acuity after KAMRA inlay implantation. J Refract Surg. 2014; 30(7): 448-453, doi: 10.3928/1081597X-20140530-03, indexed in Pubmed: 24983830.

13. Dexl AK, Jell G, Strohmaier C, et al. Long-term outcomes after monocular corneal inlay implantation for the surgical compensation of presbyopia. J Cataract Refract Surg. 2015; 41(3): 566-575, doi: 10.1016/j.jcrs.2014.05.051, indexed in Pubmed: 25726504.

14. Naroo SA, Bilkhu PS. Clinical utility of the KAMRA corneal inlay. Clin Ophthalmol. 2016; 10: 913-919, doi: 10.2147/OPTH.S89132, indexed in Pubmed: 27274194.

15. Moshirfar M, Quist TS, Skanchy DF, et al. Six-month visual outcomes for the correction of presbyopia using a small-aperture corneal inlay: single-site experience. Clin Ophthalmol. 2016; 10: 2191-2198, doi: 10.2147/OPTH.S115798, indexed in Pubmed: 27843289.

16. Igras $E, O^{\prime} C a o i m h ~ R, O ' B r i e n ~ P$, et al. Long-term Results of Combined LASIK and Monocular Small-Aperture Corneal Inlay Implantation. J Refract Surg. 2016; 32(6): 379-384, doi: 10.3928/1081597X20160317-01, indexed in Pubmed: 27304601.

17. Guilbert E, Saad A, Gatinel D. AcuTarget measurements: repeatability and comparison to OPD-Scan III. J Refract Surg. 2014; 30(3): 180-185, doi: 10.3928/1081597X-20140217-05, indexed in Pubmed: 24763722. 